\title{
Impact of photodoping on inter- and intralayer exciton emission in a $\mathrm{MoS}_{2} / \mathrm{MoSe}_{2} /$ $\mathrm{MoS}_{2}$ heterostructure
}

Cite as: Appl. Phys. Lett. 113, 062107 (2018); https://doi.org/10.1063/1.5043098

Submitted: 05 June 2018 . Accepted: 24 July 2018 . Published Online: 10 August 2018

Nan Zhang (D), Alessandro Surrente (D), Michal Baranowski, Dumitru Dumcenco ${ }^{(D)}$, Yen-Cheng Kung, Duncan K. Maude, Andras Kis, and Paulina Plochocka (D)

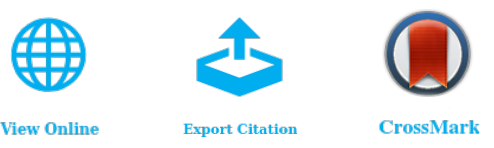

\section{ARTICLES YOU MAY BE INTERESTED IN}

Spectrally narrow exciton luminescence from monolayer $\mathrm{MoS}_{2}$ and $\mathrm{MoSe}_{2}$ exfoliated onto epitaxially grown hexagonal BN

Applied Physics Letters 113, 032106 (2018); https://doi.org/10.1063/1.5033554

Exciton diffusion in WSe 2 monolayers embedded in a van der Waals heterostructure

Applied Physics Letters 112, 152106 (2018); https://doi.org/10.1063/1.5026478

Band offsets and heterostructures of two-dimensional semiconductors

Applied Physics Letters 102, 012111 (2013); https://doi.org/10.1063/1.4774090

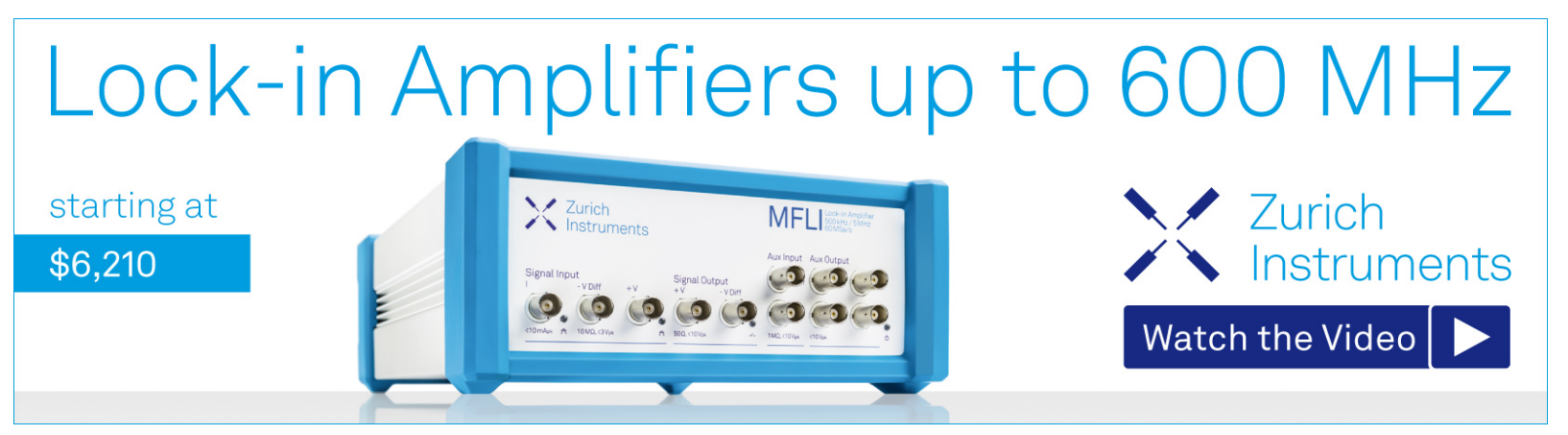




\title{
Impact of photodoping on inter- and intralayer exciton emission in a $\mathrm{MoS}_{2} / \mathrm{MoSe}_{2} / \mathrm{MoS}_{2}$ heterostructure
}

\author{
Nan Zhang, ${ }^{1}$ Alessandro Surrente, ${ }^{1}$ Michal Baranowski, ${ }^{1,2}$ Dumitru Dumcenco, ${ }^{3,4}$ \\ Yen-Cheng Kung, ${ }^{4}$ Duncan K. Maude, ${ }^{1}$ Andras Kis, ${ }^{4}$ and Paulina Plochocka ${ }^{1, a)}$ \\ ${ }^{1}$ Laboratoire National des Champs Magnétiques Intenses, UPR 3228, CNRS-UGA-UPS-INSA, \\ Grenoble and Toulouse, France \\ ${ }^{2}$ Department of Experimental Physics, Faculty of Fundamental Problems of Technology, \\ Wroclaw University of Science and Technology, Wroclaw, Poland \\ ${ }^{3}$ Department of Quantum Matter Physics, Université de Genève, 24 quai Ernest Ansermet, CH-1211 Geneva, \\ Switzerland \\ ${ }^{4}$ Electrical Engineering Institute and Institute of Materials Science and Engineering, École Polytechnique \\ Fédérale de Lausanne, CH-1015 Lausanne, Switzerland
}

(Received 5 June 2018; accepted 24 July 2018; published online 10 August 2018)

\begin{abstract}
The illumination of monolayer transition metal dichalcogenides can dynamically photoionize donor centers, increasing the concentration of free carriers. Here, we investigate the effect of such photodoping on the interlayer exciton formed across a $\mathrm{MoS}_{2} / \mathrm{MoSe}_{2} / \mathrm{MoS}_{2}$ heterostructure. We first identify the photodoping effect by monitoring the increase in the trion dissociation energy, accompanied by a characteristic tuning of the exciton/trion photoluminescence (PL) intensity ratio in $\mathrm{MoSe}_{2}$ upon exposure to laser light. At the same time, the PL intensity of the interlayer exciton significantly decreases, while the combined PL intensity of the exciton and the trion in $\mathrm{MoSe}_{2}$ is enhanced, showing that the interlayer charge transfer can be controlled by the doping level. This effect is persistent on a timescale of several hours, provided that the sample is maintained under vacuum, suggesting a mechanism involving laser induced desorption of molecules physisorbed on the surface of the heterostructure. This hypothesis is supported by the observation of a significantly faster photodoping effect when the sample is excited with a pulsed laser with the same average power. Published by AIP Publishing. https://doi.org/10.1063/1.5043098
\end{abstract}

Currently, van der Waals heterostructures are intensively investigated both from a fundamental science point of view and to overcome the limitations inherent to their constituent monolayers. $^{1,2}$ The rich choice of building blocks allows one to tailor the electronic and optical properties of the van der Waals stacks. Sandwiching monolayer transition metal dichalcogenide (TMD) between hexagonal boron nitride (hBN) dramatically improves the optical properties with a line width of the excitonic transition close to the homogeneous broadening limit, ${ }^{3,4}$ which allowed the investigation of the dark exciton fine structure. ${ }^{5}$ In addition, the excitonic properties of TMD monolayers can be conveniently tailored by an appropriate choice of the surrounding dielectric environment. ${ }^{6-9}$

van der Waals heterostructures based on semiconducting TMDs exhibit type II band alignment, ${ }^{10,11}$ which favors a charge transfer with a spatial separation of the photocreated electron-hole pairs in different layers, ${ }^{12-14}$ leading to the formation of interlayer excitons. The reduced spatial overlap of the electron-hole wave functions results in a large enhancement (by as much as five order of magnitudes) of the interlayer exciton and valley polarization lifetimes. ${ }^{15-21}$ The long radiative and valley lifetimes of interlayer excitons (tens of ns) make van der Waals heterostructures promising for their use in advanced valleytronic devices. ${ }^{2}$ To achieve this goal, a thorough understanding of the physics of the interlayer exciton is essential. Recent results demonstrate that it is possible to tune the interlayer coupling by changing the relative

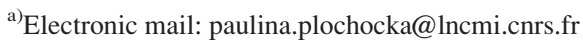

orientation of the monolayers. ${ }^{22}$ Moreover, the moiré pattern, formed due to the lattice mismatch between the two monolayers, introduces a fluctuating potential landscape for the interlayer exciton, which can influence the valley polarization. $^{23-25}$ In a gated heterostructure, the interlayer exciton valley polarization and emission intensity can be further manipulated by applying an electric field, ${ }^{20}$ suggesting a strong impact of the carrier concentration ${ }^{26,27}$ on the interlayer exciton photoluminescence (PL). Photodoping, based on the dynamic photoionization of impurities or defects, e.g., chalcogen vacancies which act as donor impurities, ${ }^{28}$ dangling bonds in the substrate or absorbants, ${ }^{28,29}$ represents an alternative way to tune the carrier concentration via optical excitation. This effect, which has been extensively investigated in monolayer TMDs, ${ }^{3,30-32}$ has not been addressed for the case of intra- and interlayer excitons in van der Waals heterostructures.

In this work, we study the influence of prolonged laser exposure on the PL of the intra- and interlayer excitons formed in a $\mathrm{MoS}_{2} / \mathrm{MoSe}_{2} / \mathrm{MoS}_{2}$ heterostructure prepared by a wet transfer method from chemical vapor deposition (CVD)-grown monolayers. ${ }^{33}$ Upon laser exposure, we observe a persistent photodoping, where the excess free carriers (electrons) tune the PL intensity ratio of the exciton and the trion of $\mathrm{MoSe}_{2}$, accompanied by an increase in $\mathrm{MoSe}_{2}$ trion dissociation energy. At the same time, the photodoping tends to reduce the PL intensity of the interlayer exciton. This is in contrast to the evolution of the $\mathrm{MoSe}_{2}$ PL intensity, with the combined integrated intensity of the exciton and trion emissions increasing with increasing 
doping level. This suggests that prolonged exposure to laser light contrasts the charge transfer between the layers, possibly due to an induced built-in electric field. This effect is sustainable on a timescale of several hours, provided that the sample is maintained under vacuum, suggesting a mechanism involving laser induced desorption of molecules (e.g., water or oxygen) physisorbed on the surface of the TMDs. Our results show that the interlayer emission and the charge transfer can be controlled by the doping level in van der Waals heterostructures.

Our $\mathrm{MoS}_{2} / \mathrm{MoSe}_{2} / \mathrm{MoS}_{2}$ heterostructures [see Fig. 1(a) for a micrograph] consist of single layer $\mathrm{MoSe}_{2}$ flakes sandwiched between single layers of $\mathrm{MoS}_{2}$. The CVD growth of the monolayers ${ }^{34,35}$ and their stacking ${ }^{33}$ have been described elsewhere. The substrate was mounted on the cold finger of a He-flow cryostat and all the measurements have been performed at $T \sim 5 \mathrm{~K}$ with the sample in vacuum. The PL was excited with a laser focused via a $50 \times$, long working distance microscope objective with a numerical aperture of 0.55 , giving an estimated spot size of $\sim 1 \mu \mathrm{m}$. We excited the PL with either a CW frequency-doubled solid-state laser emitting at $532 \mathrm{~nm}$ or the frequency-doubled output of an optical parametric oscillator, synchronously pumped by a mode-locked Ti-sapphire laser. The temporal pulse width is typically 300 fs, with a repetition rate of $80 \mathrm{MHz}$. The PL signal from the sample was collected through the same objective and detected by a spectrometer equipped with a liquid nitrogen cooled CCD camera. The laser power was measured using a powermeter placed in front of the microscope objective.

We have investigated the influence of prolonged $\mathrm{CW}$ laser illumination $(50 \mu \mathrm{W}$ power) on the emission spectrum of the $\mathrm{MoS}_{2} / \mathrm{MoSe}_{2} / \mathrm{MoS}_{2}$ trilayer by acquiring PL spectra at regular time intervals. The first spectrum was acquired immediately after the sample was exposed to light. Typical PL spectra measured after different exposure times are presented in Fig. 1(b). The spectra are characterized by three main emission peaks, attributed to the emission of the trion (T) and the neutral exciton (X) of $\mathrm{MoSe}_{2}$ and the interlayer exciton $\left(\mathrm{I}_{\mathrm{X}}\right)$ formed across the $\mathrm{MoS}_{2} / \mathrm{MoSe}_{2}$ heterostructure. ${ }^{15,21,33}$ The sharp peak observed on the low energy side of the $\mathrm{MoSe}_{2}$ trion could be related to three-dimensionally confined excitons. ${ }^{36}$ The intensity (determined by fitting with Gaussian functions) of the excitonic peaks changes over time. The intensity of the charged exciton emission increases by around $50 \%$, while the intensity of the neutral exciton emission remains more or less unchanged, as illustrated in Fig. 1(d). In contrast, the intensity of the interlayer exciton emission drops by a factor of four, as shown in Fig. 1(f). The gradual increase in the relative intensity of the trion with respect to the exciton, displayed in Fig. 1(e), points to the gradual enhancement of the free carrier concentration, due to photodoping, ${ }^{32,37}$ which increases the probability to form trions. $\mathrm{MoSe}_{2}$ and $\mathrm{MoS}_{2}$ are naturally n-doped; ${ }^{28,38,39}$ thus, the observed monotonous increase in the trion intensity over time suggests that the photocreated free carriers are electrons.

Another fingerprint of the light induced change in the carrier concentration is the temporal evolution of the trion dissociation energy, defined as the energy difference between exciton and trion emissions. This quantity is the sum of the trion binding energy $E_{\mathrm{B}}^{\mathrm{T}}$ (in the absence of other electrons) and the Fermi energy $E_{\mathrm{F}}$ measured from the bottom of the conduction band, $E_{\mathrm{X}}-E_{\mathrm{T}}=E_{\mathrm{B}}^{\mathrm{T}}+E_{\mathrm{F}}{ }^{40}$ The linear increase in the trion dissociation energy with time, shown in Fig. 1(c), reflects the upshift of the Fermi level, as the carrier density increases with time. ${ }^{30-32,37}$

In a heterostructure with type II band alignment, ${ }^{10,41}$ the observation of the negatively charged exciton emission from $\mathrm{MoSe}_{2}$ can be counterintuitive because, in the steady state, all electrons are expected to relax to $\mathrm{MoS}_{2}$. Our observations suggest that the free carriers and excitons are photoexcited simultaneously. The short radiative lifetime of intralayer excitons in TMDs, ${ }^{42-45}$ together with rapid trion formation, ${ }^{46,47}$ allows a significant proportion of the excitons and trions to recombine before the charge transfer. The presence of free electrons in $\mathrm{MoSe}_{2}$ is related to the photoionization of donors present in this material, similarly to $\mathrm{WS}_{2}$. ${ }^{30,31}$

Several mechanisms, such as light induced vacancy creation, ionization of intrinsic donors, or charges trapped by the substrate, are considered to be responsible for photodop-

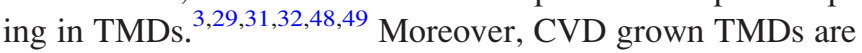
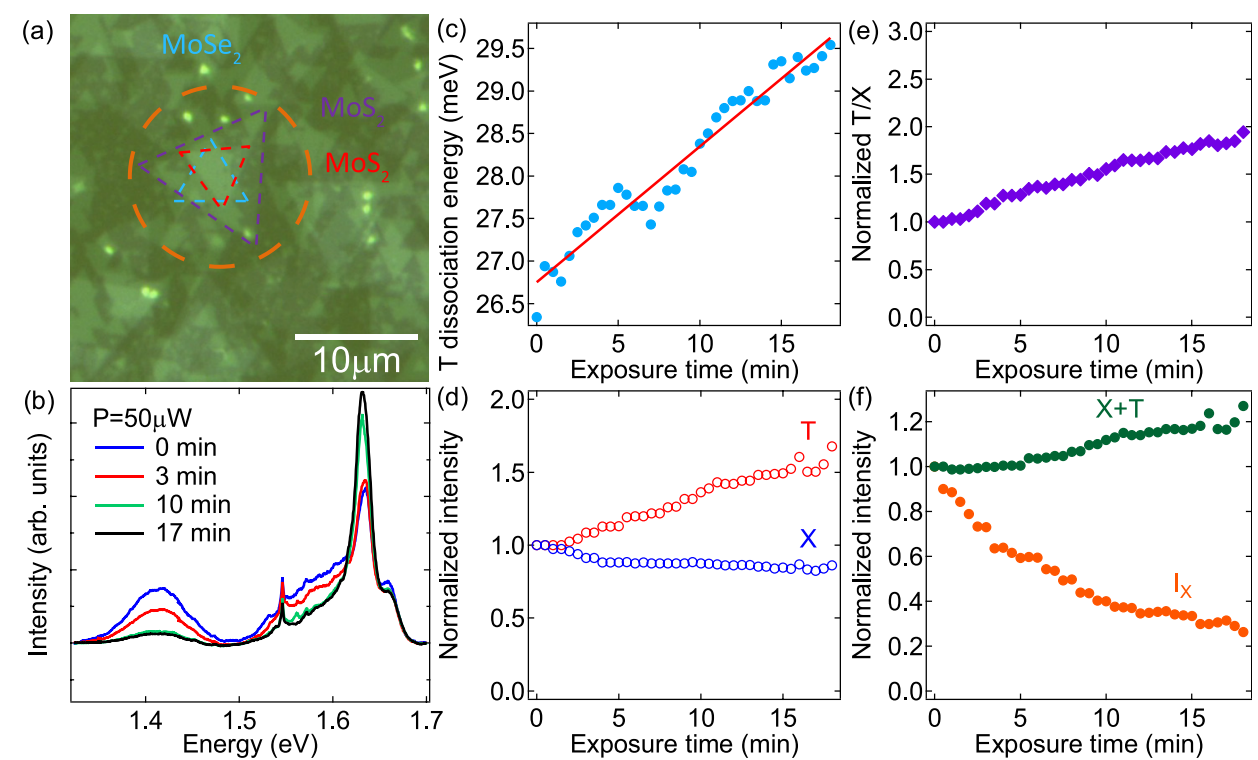

FIG. 1. (a) Micrograph of one of the investigated trilayers. (b) Low temperature PL spectra acquired after the indicated laser exposure times, showing charged ( $\mathrm{T})$ and neutral $(\mathrm{X})$ exciton emissions from $\mathrm{MoSe}_{2}$, together with emission of the interlayer exciton $\left(\mathrm{I}_{\mathrm{X}}\right)$. Temporal evolution of the (c) trion dissociation energy (the continuous line is a linear fit), (d) integrated PL intensity of the charged and neutral excitons, (e) intensity ratio of the neutral and charged excitons, and (f) combined integrated intensity of exciton $(\mathrm{X}+\mathrm{T})$ and integrated intensity of the interlayer exciton. 
known to host a large concentration of chalcogen vacancies, ${ }^{50}$ which act as donors. ${ }^{28}$ In our heterostructures, the Se vacancies in $\mathrm{MoSe}_{2}$ are partially filled by a defect-healing transfer of $\mathrm{S}$ atoms from $\mathrm{MoS}_{2}{ }^{33}$ However, the unsaturated vacancies might supply free electrons upon illumination, which leads to the observed photodoping. It is important to note that the observed changes in the sample are persistent, suggesting that prolonged exposure to laser light increases the number of photoionization centers.

The relative intensity between the neutral and charged excitons does not change even after hours in dark, provided that the sample is kept under vacuum. However, the trion-toexciton intensity ratio observed before exposure to laser light is recovered after the sample is exposed to air at room temperature. This can be explained considering the physisorption of $\mathrm{H}_{2} \mathrm{O}$ and $\mathrm{O}_{2}$ molecules to TMDs. These two species bind to the chalcogen vacancies with a binding energy of $\sim 100 \mathrm{meV}$ and deplete the naturally n-doped TMDs of electrons. $^{29,48}$ Upon laser illumination, photogenerated holes neutralize the adsorbed species, leading to their photodesorption. ${ }^{51,52}$ This explains the gradual increase in the free carrier concentration, which leads to the behavior summarized in Fig. 1.

In Fig. 1(f), we plot the combined integrated intensity of the charged and neutral excitons $(\mathrm{X}+\mathrm{T})$ as a function of time. In contrast to isolated monolayers, ${ }^{3,30}$ the PL intensity of intralayer excitonic species slightly increases with increasing doping. This is consistent with a reduced charge transfer rate with increasing doping level, which also accounts for the decrease in the interlayer exciton emission intensity with increasing doping. This effect might be exploited to optically control the efficiency of interlayer charge transfer by tuning the doping level in TMD-based van der Waals heterostructures. The relative weight of the intralayer to interlayer emission intensity can also be controlled electrostatically, as demonstrated in a p-n junction consisting of a TMD heterobilayer. ${ }^{53}$

We tentatively ascribe the decreasing charge transfer efficiency to the creation of a built-in electric field, possibly linked with the desorption of water and oxygen molecules from the surface of the TMD stack. This scenario is also consistent with the observed blue shift of the PL peak energy of the interlayer exciton measured at high excitation power $(P=200 \mu \mathrm{W})$. A comparison of the PL spectrum of the interlayer exciton at the beginning and at the end of the exposure to the laser light is shown in Fig. 2(a), where the $\sim 6 \mathrm{meV}$ blue shift is highlighted. The global temporal dependence of the energy of the interlayer exciton transition, determined using the center of mass approach, ${ }^{54}$ which yields results equivalent to Gaussian fitting, ${ }^{21}$ is illustrated in Fig. 2(b). This trend is similar to the observed blue shift of the indirect exciton transition in type II quantum wells with increasing excitation power, ${ }^{55,56}$ which results from large electric fields generated at high carrier concentrations. It is also in agreement with the recently observed drop in the emission intensity of the interlayer excitons in gated heterostructures both for $\mathrm{n}$ - and p-type doping. ${ }^{57}$

Pulsed excitation has been reported to have a dramatic impact on the PL of intralayer excitons, with a rapid and complete suppression of the intralayer exciton emission accompanied by a radical modification of its line shape. ${ }^{3}$ To
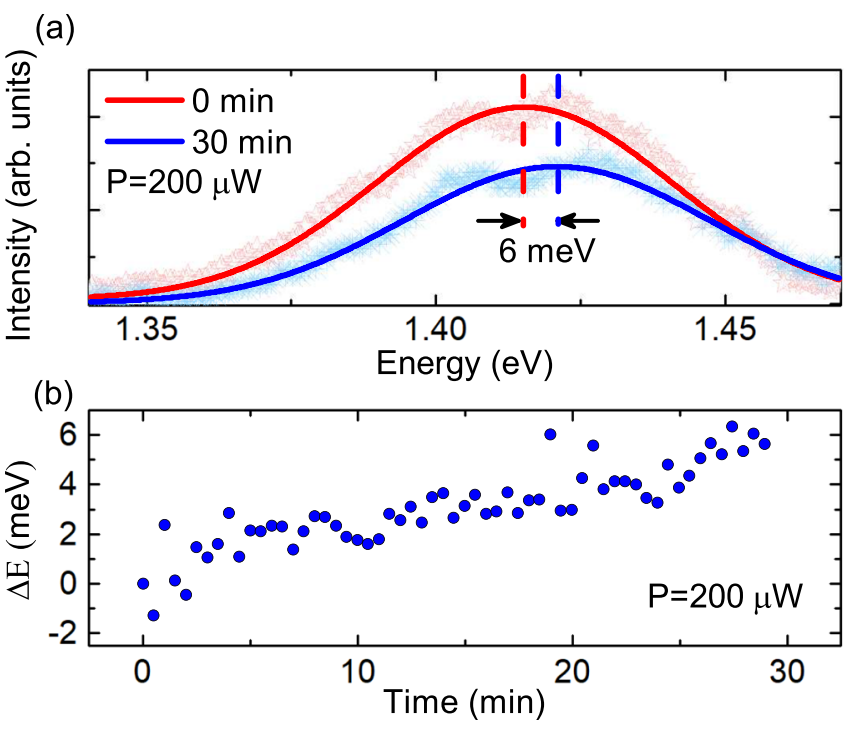

FIG. 2. (a) PL spectra of the interlayer exciton at the beginning and end of the exposure to a high power laser. (b) Temporal evolution of the energy of the interlayer exciton PL at high excitation power.

investigate the effects of different excitation conditions on the PL intensity of the interlayer exciton we monitored its temporal evolution exciting at 25 and $200 \mu \mathrm{W}$ average power, using both $\mathrm{CW}$ and pulsed lasers. In Fig. 3, we show the temporal evolution of the PL intensity of the interlayer exciton. While its PL intensity drops only marginally when we excite with a CW laser at $25 \mu \mathrm{W}$, the effects of high excitation power are more prominent. Pulsed excitation suppresses the PL of the interlayer exciton more efficiently: an average power of $200 \mu \mathrm{W}$ almost completely quenches the interlayer exciton emission after an exposure of $\sim 30 \mathrm{~min}$. The peak power of the pulsed laser $(\simeq 830 \mathrm{~W}$ for $200 \mu \mathrm{W}$ average power) creates a large density of carriers, which leads to more efficient photodesorption ${ }^{51,52}$ of the physisorbed gas molecules.

In conclusion, we have investigated the effects of persistent photodoping on the exciton emission in a $\mathrm{MoS}_{2} / \mathrm{MoSe}_{2} /$ $\mathrm{MoS}_{2}$ heterostructure. Prolonged exposure to laser light increases the number of photoionization centers present, persistently increasing the photodoping effect (number of dynamically photoionized electrons in the conduction band), provided that the sample is kept under vacuum. This suggests

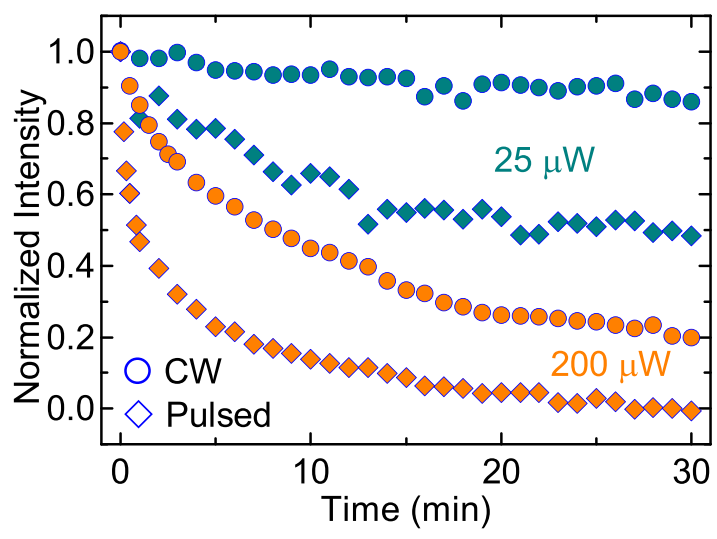

FIG. 3. Temporal evolution of the PL intensity of the interlayer exciton at different excitation powers under both $\mathrm{CW}$ and pulsed excitation. 
that the effect is linked with laser induced desorption of water or oxygen molecules from the surface. The impact of the free carrier concentration on the interlayer emission might represent a possible step towards the control of the charge transfer rate in van der Waals heterostructures. If a more stable electrostatic environment is desired, doping following laser illumination can be prevented by encapsulating the van der Waals heterostructure in $\mathrm{hBN}{ }^{58}$

This work was partially supported by the Région MidiPyrénées under Contract No. MESR 13053031, BLAPHENE, and STRABOT projects, which received funding from the IDEX Toulouse, Emergence Program, by "Programme Investissements d'Avenir" under Program No. ANR-11IDEX-0002-02, reference ANR-10-LABX-0037-NEXT. N.Z. holds a fellowship from the Chinese Scholarship Council (CSC). This work was also financially supported by the Swiss SNF Sinergia Grant No. 147607. M.B. acknowledges support from the Polish Ministry of Higher Education and Science through Grant No. DEC 1648/MOB/V/2017/0.

${ }^{1}$ A. K. Geim and I. V. Grigorieva, Nature 499, 419 (2013).

${ }^{2}$ J. R. Schaibley, H. Yu, G. Clark, P. Rivera, J. S. Ross, K. L. Seyler, W. Yao, and X. Xu, Nat. Rev. Mater. 1, 16055 (2016).

${ }^{3}$ F. Cadiz, E. Courtade, C. Robert, G. Wang, Y. Shen, H. Cai, T. Taniguchi, K. Watanabe, H. Carrere, D. Lagarde, M. Manca, T. Amand, P. Renucci, S. Tongay, X. Marie, and B. Urbaszek, Phys. Rev. X 7, 021026 (2017).

${ }^{4}$ O. A. Ajayi, J. V. Ardelean, G. D. Shepard, J. Wang, A. Antony, T. Taniguchi, K. Watanabe, T. F. Heinz, S. Strauf, X.-Y. Zhu, and J. C. Hone, 2D Mater. 4, 031011 (2017).

${ }^{5}$ C. Robert, T. Amand, F. Cadiz, D. Lagarde, E. Courtade, M. Manca, T. Taniguchi, K. Watanabe, B. Urbaszek, and X. Marie, Phys. Rev. B 96, 155423 (2017).

${ }^{6}$ S. Borghardt, J.-S. Tu, F. Winkler, J. Schubert, W. Zander, K. Leosson, and B. E. Kardynał, Phys. Rev. Mater. 1, 054001 (2017).

${ }^{7}$ A. Raja, A. Chaves, J. Yu, G. Arefe, H. M. Hill, A. F. Rigosi, T. C. Berkelbach, P. Nagler, C. Schueller, T. Korn, C. Nuckolls, J. Hone, L. E. Brus, T. F. Heinz, D. R. Reichman, and A. Chernikov, Nat. Commun. 8, 15251 (2017)

${ }^{8}$ P. Steinleitner, P. Merkl, A. Graf, P. Nagler, K. Watanabe, T. Taniguchi, J. Zipfel, C. Schüller, T. Korn, A. Chernikov, S. Brem, M. Selig, G. Berghäuser, E. Malic, and R. Huber, Nano Lett. 18, 1402 (2018).

${ }^{9}$ M. Florian, M. Hartmann, A. Steinhoff, J. Klein, A. W. Holleitner, J. J. Finley, T. O. Wehling, M. Kaniber, and C. Gies, Nano Lett. 18, 2725 (2018).

${ }^{10}$ J. Kang, S. Tongay, J. Zhou, J. Li, and J. Wu, Appl. Phys. Lett. 102, 012111 (2013).

${ }^{11}$ S. Latini, K. T. Winther, T. Olsen, and K. S. Thygesen, Nano Lett. 17, 938 (2017).

${ }^{12}$ X. Hong, J. Kim, S.-F. Shi, Y. Zhang, C. Jin, Y. Sun, S. Tongay, J. Wu, Y. Zhang, and F. Wang, Nat. Nanotechnol. 9, 682 (2014).

${ }^{13}$ F. Ceballos, M. Z. Bellus, H.-Y. Chiu, and H. Zhao, ACS Nano 8, 12717 (2014).

${ }^{14}$ H. Chen, X. Wen, J. Zhang, T. Wu, Y. Gong, X. Zhang, J. Yuan, C. Yi, J. Lou, P. M. Ajayan, W. Zhuang, G. Zhang, and J. Zheng, Nat. Commun. 7, 12512 (2016).

${ }^{15}$ M. Baranowski, A. Surrente, L. Klopotowski, J. Urban, N. Zhang, D. K. Maude, K. Wiwatowski, S. Mackowski, Y.-C. Kung, D. Dumcenco, A. Kis, and P. Plochocka, Nano Lett. 17, 6360 (2017).

${ }^{16} \mathrm{M}$. N. Brunetti, O. L. Berman, and R. Y. Kezerashvili, J. Phys.: Condens. Matter 30, 225001 (2018).

${ }^{17}$ B. Miller, A. Steinhoff, B. Pano, J. Klein, F. Jahnke, A. Holleitner, and U. Wurstbauer, Nano Lett. 17, 5229 (2017).

${ }^{18}$ P. Nagler, M. V. Ballottin, A. A. Mitioglu, F. Mooshammer, N. Paradiso, C. Strunk, R. Huber, A. Chernikov, P. C. Christianen, C. Schüller, and T. Korn, Nat. Commun. 8, 1551 (2017).

${ }^{19}$ P. Rivera, J. Schaibley, A. Jones, J. Ross, S. Wu, G. Aivazian, P. Klement, K. Seyler, G. Clark, N. Ghimire, J. Yan, M. DG, W. Yao, and X. Xu, Nat. Commun. 6, 6242 (2015).
${ }^{20}$ P. Rivera, K. L. Seyler, H. Yu, J. R. Schaibley, J. Yan, D. G. Mandrus, W. Yao, and X. Xu, Science 351, 688 (2016).

${ }^{21}$ A. Surrente, Ł. Klopotowski, N. Zhang, M. Baranowski, A. Mitioglu, M. V. Ballottin, P. C. Christianen, D. Dumcenco, Y.-C. Kung, D. K. Maude, A. Kis, and P. Plochocka, Nano Lett. 18, 3994 (2018).

${ }^{22}$ P. K. Nayak, Y. Horbatenko, S. Ahn, G. Kim, J.-U. Lee, K. Y. Ma, A.-R. Jang, H. Lim, D. Kim, S. Ryu, H. Cheong, N. Park, and H. S. Shin, ACS Nano 11, 4041 (2017).

${ }^{23}$ J. Kang, J. Li, S.-S. Li, J.-B. Xia, and L.-W. Wang, Nano Lett. 13, 5485 (2013).

${ }^{24}$ F. Wu, T. Lovorn, and A. H. MacDonald, Phys. Rev. B 97, 035306 (2018).

${ }^{25}$ C. Zhang, C.-P. Chuu, X. Ren, M.-Y. Li, L.-J. Li, C. Jin, M.-Y. Chou, and C.-K. Shih, Sci. Adv. 3, e1601459 (2017).

${ }^{26}$ J. S. Ross, S. Wu, H. Yu, N. J. Ghimire, A. M. Jones, G. Aivazian, J. Yan, D. G. Mandrus, D. Xiao, W. Yao, and X. Xu, Nat. Commun. 4, 1474 (2013).

${ }^{27}$ K. F. Mak, K. He, C. Lee, G. H. Lee, J. Hone, T. F. Heinz, and J. Shan, Nat. Mater. 12, 207 (2013).

${ }^{28}$ C.-P. Lu, G. Li, J. Mao, L.-M. Wang, and E. Y. Andrei, Nano Lett. 14, 4628 (2014)

${ }^{29}$ S. Tongay, J. Suh, C. Ataca, W. Fan, A. Luce, J. S. Kang, J. Liu, C. Ko, R. Raghunathanan, J. Zhou, F. Ogletree, J. Li, J. C. Grossman, and J. Wu, Sci. Rep. 3, 2657 (2013).

${ }^{30}$ M. Currie, A. Hanbicki, G. Kioseoglou, and B. Jonker, Appl. Phys. Lett. 106, 201907 (2015).

${ }^{31}$ A. Mitioglu, P. Plochocka, J. Jadczak, W. Escoffier, G. Rikken, L. Kulyuk, and D. Maude, Phys. Rev. B 88, 245403 (2013).

${ }^{32}$ N. Lundt, E. Cherotchenko, O. Iff, X. Fan, Y. Shen, P. Bigenwald, A. Kavokin, S. Höfling, and C. Schneider, Appl. Phys. Lett. 112, 031107 (2018).

${ }^{33}$ A. Surrente, D. Dumcenco, Z. Yang, A. Kuc, Y. Jing, T. Heine, Y.-C. Kung, D. K. Maude, A. Kis, and P. Plochocka, Nano Lett. 17, 4130 (2017).

${ }^{34}$ D. Dumcenco, D. Ovchinnikov, K. Marinov, P. Lazic, M. Gibertini, N. Marzari, O. L. Sanchez, Y.-C. Kung, D. Krasnozhon, M.-W. Chen, S. Bertolazzi, P. Gillet, A. Fontcuberta i Morral, A. Radenovic, and A. Kis, ACS Nano 9, 4611 (2015).

${ }^{35}$ A. Mitioglu, K. Galkowski, A. Surrente, L. Klopotowski, D. Dumcenco, A. Kis, D. Maude, and P. Plochocka, Phys. Rev. B 93, 165412 (2016).

${ }^{36}$ A. Srivastava, M. Sidler, A. V. Allain, D. S. Lembke, A. Kis, and A. Imamoğlu, Nat. Nanotechnol. 10, 491 (2015).

${ }^{37}$ F. Cadiz, C. Robert, G. Wang, W. Kong, X. Fan, M. Blei, D. Lagarde, M. Gay, M. Manca, T. Taniguchi, K. Watanabe, T. Amand, X. Marie, P. Renucci, S. Tongay, and B. Urbaszek, 2D Mater. 3, 045008 (2016).

${ }^{38}$ B. Radisavljevic, A. Radenovic, J. Brivio, I. V. Giacometti, and A. Kis, Nat. Nanotechnol. 6, 147 (2011).

${ }^{39}$ B. W. Baugher, H. O. Churchill, Y. Yang, and P. Jarillo-Herrero, Nano Lett. 13, 4212 (2013).

${ }^{40}$ V. Huard, R. Cox, K. Saminadayar, A. Arnoult, and S. Tatarenko, Phys. Rev. Lett. 84, 187 (2000).

${ }^{41}$ C. Gong, H. Zhang, W. Wang, L. Colombo, R. M. Wallace, and K. Cho, Appl. Phys. Lett. 103, 053513 (2013).

${ }^{42}$ D. Lagarde, L. Bouet, X. Marie, C. Zhu, B. Liu, T. Amand, P. Tan, and B. Urbaszek, Phys. Rev. Lett. 112, 047401 (2014).

${ }^{43}$ C. Robert, D. Lagarde, F. Cadiz, G. Wang, B. Lassagne, T. Amand, A. Balocchi, P. Renucci, S. Tongay, B. Urbaszek, and X. Marie, Phys. Rev. B 93, 205423 (2016).

${ }^{44}$ T. Godde, D. Schmidt, J. Schmutzler, M. Aßmann, J. Debus, F. Withers, E. Alexeev, O. Del Pozo-Zamudio, O. Skrypka, K. Novoselov, M. Bayer, and A. Tartakovskii, Phys. Rev. B 94, 165301 (2016).

${ }^{45}$ T. Korn, S. Heydrich, M. Hirmer, J. Schmutzler, and C. Schüller, Appl. Phys. Lett. 99, 102109 (2011).

${ }^{46}$ A. Singh, G. Moody, K. Tran, M. E. Scott, V. Overbeck, G. Berghäuser, J. Schaibley, E. J. Seifert, D. Pleskot, N. M. Gabor, J. Yan, D. G. Madrus, M. Richter, E. Malic, X. Xu, and X. Li, Phys. Rev. B 93, 041401 (2016).

${ }^{47}$ K. Hao, L. Xu, P. Nagler, A. Singh, K. Tran, C. K. Dass, C. Schuller, T. Korn, X. Li, and G. Moody, Nano Lett. 16, 5109 (2016).

${ }^{48}$ S. Tongay, J. Zhou, C. Ataca, J. Liu, J. S. Kang, T. S. Matthews, L. You, J. Li, J. C. Grossman, and J. Wu, Nano Lett. 13, 2831 (2013).

${ }^{49}$ M. M. Furchi, D. K. Polyushkin, A. Pospischil, and T. Mueller, Nano Lett. 14, 6165 (2014).

${ }^{50}$ H.-V. Han, A.-Y. Lu, L.-S. Lu, J.-K. Huang, H. Li, C.-L. Hsu, Y.-C. Lin, M.-H. Chiu, K. Suenaga, C.-W. Chu, H.-C. Kuo, W.-H. Chang, L.-J. Li, and Y. Shi, ACS Nano 10, 1454 (2016). 
${ }^{51}$ W. Zhang, J.-K. Huang, C.-H. Chen, Y.-H. Chang, Y.-J. Cheng, and L.-J. Li, Adv. Mater. 25, 3456 (2013).

${ }^{52}$ Y. Lee, J. Yang, D. Lee, Y.-H. Kim, J.-H. Park, H. Kim, and J. H. Cho, Nanoscale 8, 9193 (2016).

${ }^{53}$ J. S. Ross, P. Rivera, J. Schaibley, E. Lee-Wong, H. Yu, T. Taniguchi, K. Watanabe, J. Yan, D. Mandrus, D. Cobden, W. Yao, and X. Xu, Nano Lett. 17, 638 (2017).

${ }^{54}$ G. Aivazian, Z. Gong, A. M. Jones, R.-L. Chu, J. Yan, D. G. Mandrus, C. Zhang, D. Cobden, W. Yao, and X. Xu, Nat. Phys. 11, 148 (2015).
${ }^{55}$ S. Zaitsev, A. Maksimov, I. Tartakovskii, D. Yakovlev, M. Bayer, and A. Waag, Phys. Rev. B 76, 035312 (2007).

${ }^{56}$ M. Baranowski, M. Syperek, R. Kudrawiec, J. Misiewicz, J. Gupta, X. Wu, and R. Wang, Appl. Phys. Lett. 98, 061910 (2011).

${ }^{57}$ A. Ciarrocchi, D. Unuchek, A. Avsar, K. Watanabe, T. Taniguchi, and A. Kis, preprint arXiv:1803.06405 (2018).

${ }^{58}$ E. Courtade, B. Han, S. Nakhaie, C. Robert, X. Marie, P. Renucci, T. Taniguchi, K. Watanabe, L. Geelhaar, J. Lopes, and B. Urbaszek, Appl. Phys. Lett. 113, 032106 (2018). 